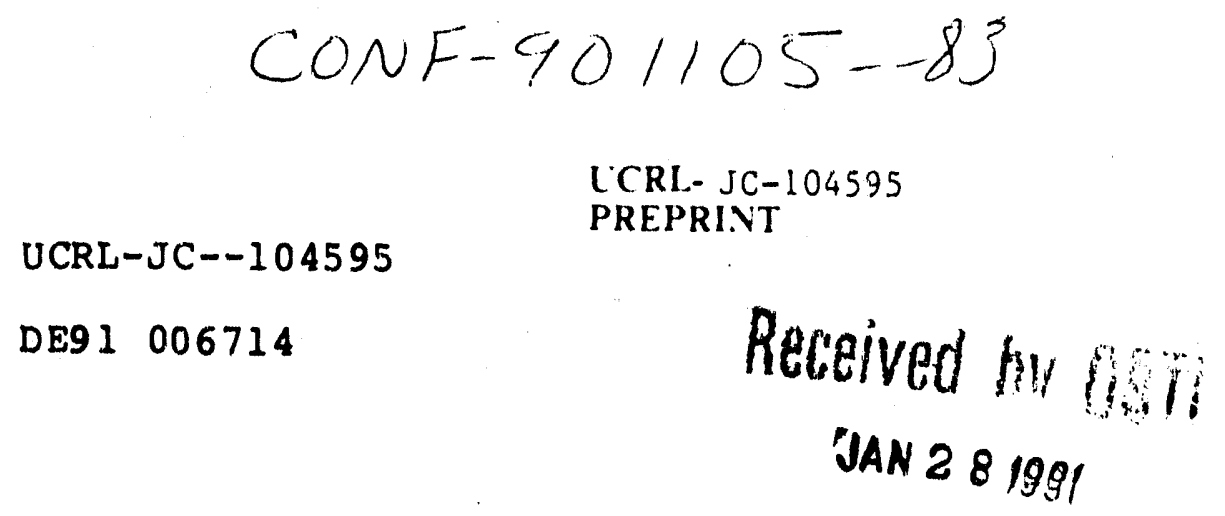

\title{
Defect Dependence of Positron Lifetî̀mes in Oxide Superconductors
}

P. A. Sterne
J. C. O'Brien
R. H. Howell
J. H. Kaiser

This paper was prepared for submission to Proceedings, MRS Symposium K--Defects in Materials Materials Kesearch Society Meeting Boston, MA

November $26-30 / 1990$

November, 1990

This is a preprint of a paper intended for publication in a journal or proceedings. Since clianges may be made before publication, this preprint is made available with the understanding that it will nat be cited or reproduced without the permission of the author. 
This document was prepared as an account of work sponsored by an arency at the United Stales Government. Neither the United States Corernment nor the University of Californiz nor any of their employees makes any warranty, express or implied, or assumes any legal liability of responsibility for the accuracy, completeness, of usefulness of ans information, apparatus, product, or process disclosed, or represents that its use would not infringe privately owned rights. Reference herein to any specific commercial products, process, or service by irade name, trademark, manufacturer, or olherwise. does nol necessarily constifute or imply its endorsement, recommendation. or favoring by the United States Government or the University of Californiz. The views and opinions of authors expressed herein do not mecessarily state of reflext those of the United States Government or the University of California, and shall not be used for advertising or product endorsement purposes. 


\title{
DEFECT DEPENDENCE OF POSITRON LIFETIMES IN OXIDE SUPERCONDUCTORS
}

\author{
P.A. STERNE*, J.C. O'BRIEN', R.H. HOWELL' and J.H. KAISER ${ }^{\dagger}$ \\ - Lawrence Livermore National Laboratory, Livermore, CA 94550 \\ 'Department of Physics, University of Texas at Arlington, TX 76019
}

\begin{abstract}
First principles calculations of the positron lifetimes are used to interpret experimental lifetime data for $\mathrm{Ba}_{1-x} \mathrm{~K}_{x} \mathrm{BiO}_{3}$. The observed lifetimes are found to be very sensitive to the presence of defects in the sample. The temperature dependence of the lifetime and the change in lifetime with sample aging are both attributed to defects. The qualitative differences between metal vacancy defects and oxygen vacancy defects in metal oxides are also discussed.
\end{abstract}

\section{INTRODUCTION}

Positron lifetime experiments provide a very sensitive probe of the defect structure in materials, $\{1,2]$ since the positively charged positrons are strongly repelled by the atomic nuclei, and so tend to favor regions with vacancies and other defects. Positrons are trapped at these defects and annihilate with electrons there. The annihilation rate, the inverse of the lifetime, depends on the electron charge density sampled by the positron, and so different defects have their own characteristic lifetimes. In some cases, the experimental spectra provide clear evidence of the influence of defect trapping on the positrons, such as a strong temperature dependence in the measured lifetime. In other cases, the spectrum may be almost temperature independent, and apparently characteristic of the bulk, defect-free lifetime, when, in fact, the annihilation rate is dominated by trapping at defects. One of the major problems with this experimental technique is determining when the measured lifetimes are defect rather than bulk related, and which defecis are responsible for the observed lifetime, or range of lifetimes.

In this paper we present a theoretical study of positron lifetimes in on $\mathrm{Ba}_{1-x} \mathrm{~K}_{x} \mathrm{BiO}_{3}$ (BKBO) and compare our calculations with experimental data for samples with $x=0.4$ and 0.5. Details of the experiment are presented in an accompanying paper.[3] We use first principles calculations of the annihilation rate to deduce what the positron lifetime should be for bulk, defect-free $\mathrm{BKBO}$. We also compute the range of lifetimes expected for some simple monovacancies, and use these calculations to interpret the experimental data. The calculations indicate that values of lifetimes consistent with experiment are only obtained when we consider positron lifetimes at defect sites. This is consistent with the observed lifetime dependence on sample aging, which is presumed to be due to a rearrangement of these defects over time. The different effects of metal and oxygen vacancies are also discussed.

\section{METHOD}

The theoretical calculations have been performed using the method described in detail in Ref. [4]. This is a first principles approach for calculating the positron lifetime in solids, based on the Linear Muffin Tin Orbital method.[5] Electron-electron and electron-positron interactions are included through a Local Density Approximation approach. The method 
involves no fitting to experimental positron lifetime data, and gives excellent agreement with experiment for a wide range of elemental metals and metal vacancies. [4]

A lattice constant of $4.28 \AA$ was used. Since the experimental data were taken on samples in the simple cubic perorskite phase, this structure and lattice constant was used for all the calculations, throughout the entire range of potassium concentrations. Increasing the lattice constant by $1 \%$ increases the lifetime by only $3 \mathrm{ps}$, which is small compared to the lifetime differences we will be interested in, ald so the change in lattice constant with $K$ concentration can be ignored. A superceli geometry was used to perform calculations to represent alloying on the $\mathrm{K} / \mathrm{Ba}$ sublatice and the presence of vacancies. A 10 atom supercell with an fcc lattice, corresponding to a doubling of the primitive cell, and a 20 atom supercell, four times the size of the primitive cell, with a bcc lattice were considered. Relaxations around the vacancy sites were neglected. Since a supercell approach is used, we cannot calculate the lifetime for arbitrary potassium concentrations. However, the results for $x=0.0,0.25,0.5$ and 1.0 show that the lifetime increases monotonically with potassium concentration. The end-points, $x=0$ and $x=1$ therefore provide an appropriate range within which we expect the experimental lifetime to fall for arbitrary potassium concentration. The calculations were performed with atomic sphere sizes of $4.286 \mathrm{a}$.u. for barium and potassium, 2.5 a.u. for bismuth and 2.2 a.u. for oxygen. Significantly different sphere sizes (4.448 a.u., 2.75 a.u. and 1.8 a.u. respectively) yielded essentially identical results for the stoichiometric compounds, indicating that the lifetime calculations are not sensitive to the choice of sphere sizes.

\section{RESULTS AND DISCUSSION}

Figure 1 shows the experimental data for the lifetimes on two BKBO samples, with $x=0.4,0.5$. The experimental lifetime values are in the range of $210-250 \mathrm{ps}$, which is to be compared with theoretical calculations for bulk $\mathrm{BaBiO}_{3}$ of $168 \mathrm{ps}$ and bulk $\mathrm{KBaBiO}_{3}$ at 193ps. Supercell calculations for $x=0.25$ and 0.5 fall between the two theoretical bulk calculations, so it is clear that there is a large discrepancy between the calculated bulk lifetimes and the measured experimental results. The experimental data shows a number of features which are suggestive of the influence of defects on the positron lifetime, however, so we may not expect the measured lifetime to correspond to a theoretical calculation for a perfectly periodic system. The temperature dependence of the lifetime, particularly in $x=0.5$ sample, is much too large to attribute to thermal lattice expansion. Such a temperature dependence can be caused by a competition between two defect states which can trap the positron. At low temperatures, the positron readily falls into a shallow defect state and stays there until it annihilates. As the temperature increases, the positron can gain enough thermal energy to escape from the shallow trap, and fall into a deeper trap before annihilating. Since sites that attract positrons are generally not attractive for the electrons, the charge density overlap with the positron is usually lower in the deeper trap. This leads to a reduction in the annihilation rate, which depends on the overlap of the electron and positron charge densities, and so the lifetime increases. This model gives a qualitative explanation for the observed increase in the positron lifetime with temperature.

The $x=0.4$ data is somewhat less temperature dependent. This might suggest that the sample is defect free, and that the bulk lifetime should correspond to one or other of these lifetimes, but this is not the case. The large change in lifetime with aging suggests that the

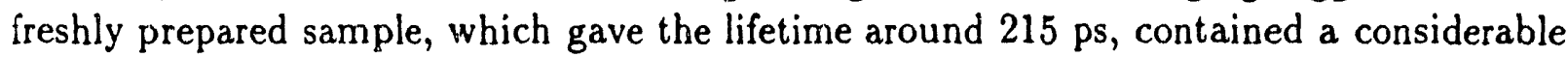
number of defects, some of which annealed out during the aging process. The aging was carried out at room temperature, so it is unlikely that any new defects were created during this process. Instead, the existing defects underwent some form of rearrangement, and some 


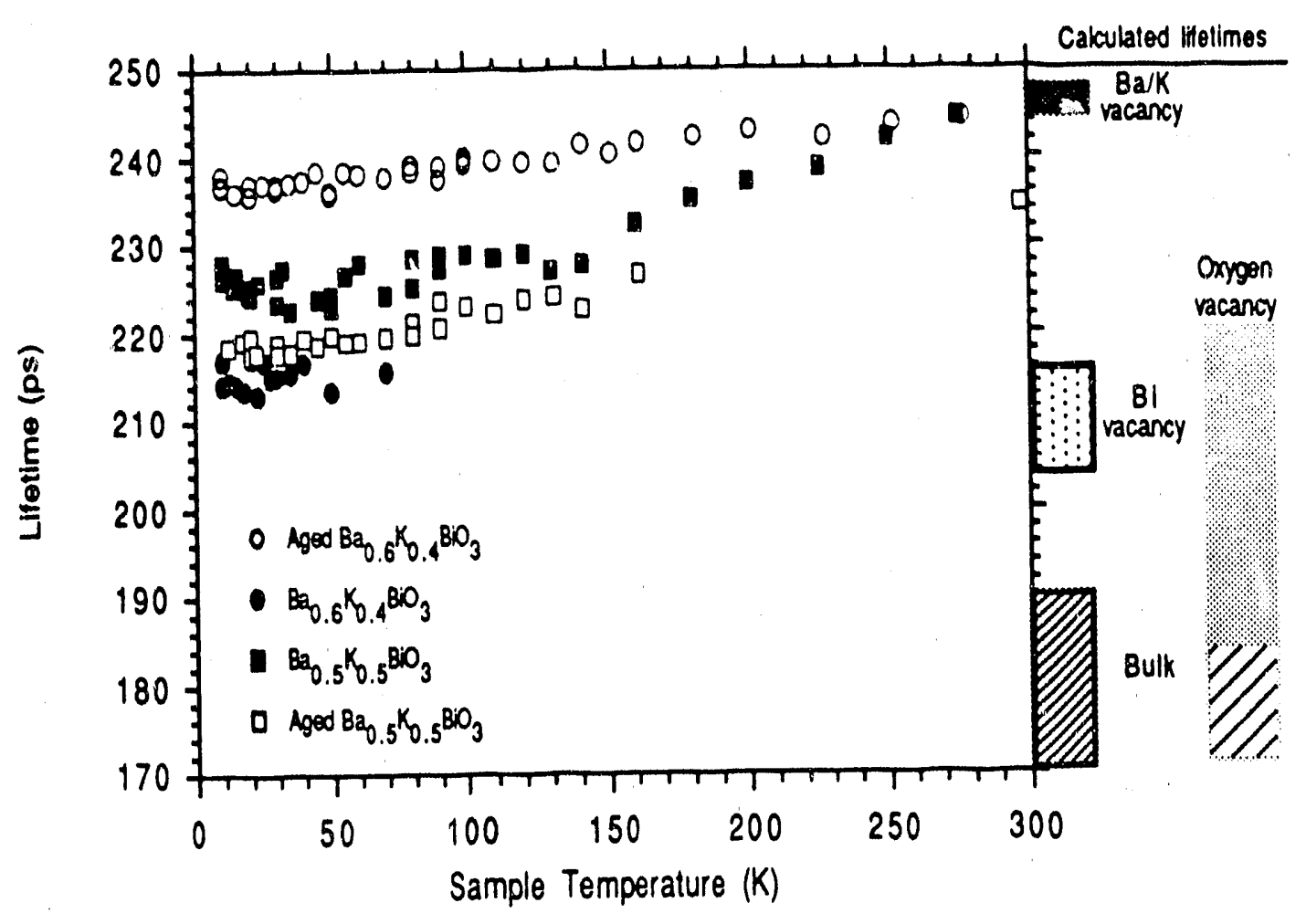

Figure 1: Experimental lifetimes for $\mathrm{Ba}_{1-x} \mathrm{~K}_{x} \mathrm{BiO}_{3}$. Unaged data were taken on freshly prepared melt processed samples. Aged data were on the same samples after leaving in air at room temperature for about one year. Ranges of lifetimes calculated theoretically for the bulk and vacancy defects are shown on the right hand side. The range in each case corresponds to the extreme limits of potassium concentration, from $x=0$ to $x=1$.

of them were compensated. The remaining defects exhibit a lifetime which is essentially the same as the high temperature lifetime of the $x=0.5$ material, so it is likely that they are due to the same native defect.

The range of calculated lifetimes for bulk BKBO and some simple monovacancy defects are shown on the right hand side of Figure 1. The calculated lifetimes depend on the potassium concentration, with longer values for higher potassium concentrations, so the bars in Figure 1 cover potassium concentrations from $x=0$ to $x=1$. The $\mathrm{Ba}$ and $\mathrm{K}$ vacancy lifetimes are almost independent of potassium concentration and their value of around 248ps closely matches the highest lifetimes measured in hoth samples. The Bi vacancy shows a somewhat stronger potassium dependence, suggesting that the positron is not trapped so deeply in a Bi vacancy and that the positron wavefunction may still have some overlap with the surrounding charge density. This more shallow defect has lifetimes in the range of $205-215 \mathrm{ps}$, which tie in reasnilably well with the lower values of the lifetime measured in the experiments. The calculations indicate that positron trapping at these two vacancy sites can account both qualitatively and quantitatively for the observed lifetime behavior. At low temperatures, the positron has a higher probability of entering the shallow Bi vacancy site. At higher temperatures, it is no longer trapped at the Bi vacancy and so traps at the deeper $\mathrm{Ba}$ or $\mathrm{K}$ vacancy, thereby leading to an increase in the observed lifetime. 


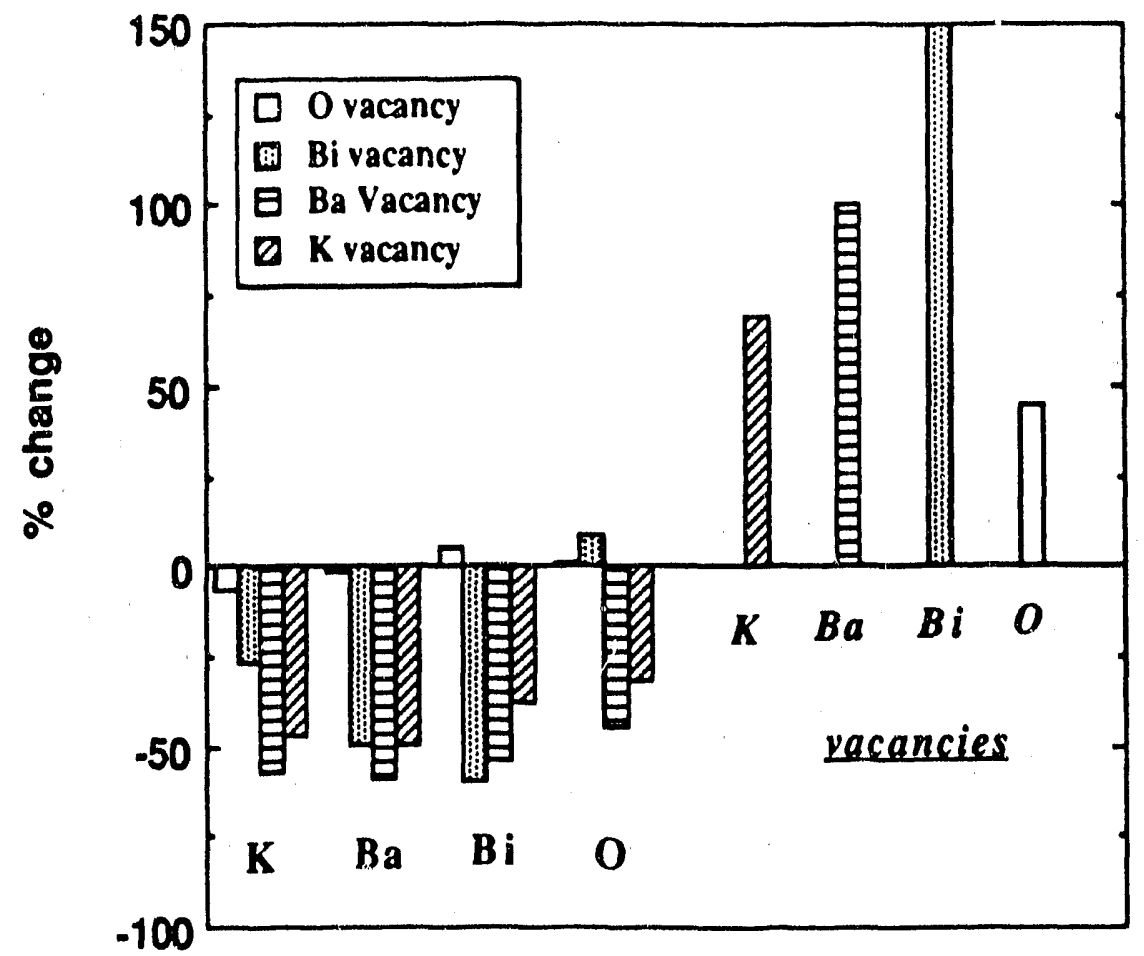

Figure 2: Change in positron charge density on the various atomic sites when vacancies are created. The four bars on the right hand side of the graph show the increase in positron rensity on the vacancy compared with the density on that site in a bulk calculation. The sets of bars on the left hand side show the change in the density induced on the neighboring atomic sites when a vacancy of a given type is created. For example, the horizontally striped bar shows the change in the positron density on all the other atoms when a vacancy is created on one of the barium sites.

If the temperature dependence were due to competition between just two defects, each with its own characteristic lifetime, the experimental analysis of the data would have obtained a fit with two distinct sample components to the lifetime. In fact, the best fit in all cases came with a single sample lifetime component[3]. This suggests that the $\mathrm{Bi}$ vacancy, or some other defect which we have not considered here, may exist in a range of environments, with different potassium concentrations and oxygen vacancies, giving rise to a range of possible lifetimes which cannot be resolved experimentally beyond a single, average value. In such a case, the temperature dependence would show a gradual rise from a low lifetime value toward: the longer lifetime characteristic of the deeper trap, as is seen experimentally.

The metal vacancies tend to trap the positron and produce a dramatic change in the positron charge density on all atomic sites, including the vacancy site. The oxygen vacancies behave rather differently. The electrostatic potential of the crystal favors a positive ion at the metal atom site, so the positively charged positron is naturally attracted to a metal atom vacancy. This potential favors a negatively charged ion on the oxygen site, however, so the positron is less likely to favor an oxygen vacancy. This is shown in Figure 2 where we plot the calculated change in positron wavefunction on each site in the lattice when 
different vacancies are included. There are large changes in the positron distribution on all sites when the metal vacancies are introduced, as indicated by the shaded bars on the left-hand side of the graph. The metal atom vacancy has a strong effect on the positron, localizing it at the defect and removing it from neighboring atoms. The oxygen vacancy, however, introduces very little change in the positron distribution on the neighboring sites, indicating that the average potential that the positron sees on an oxygen atom and an oxygen vacancy are very similar. It should be noted that the electron charge densities were calculated self-consistently for the vacancies, and that different results would be obtained if we did not allow the electron charge density to relax into the oxygen vacancy.

Oxygen vacancies do not perturb the positron wavefunction significantly, but they do lead to an increase in the lifetime, due to the reduction in the electron charge density on a vacancy site compared with an occupied oxygen site. The lowest lifetime we calculated for oxygen vacancy structures was $183 \mathrm{ps}$ for $\mathrm{Ba}_{2} \mathrm{Bi}_{2} \mathrm{O}_{5}$, or one vacancy for every six oxygen sites. This is indicated in the shaded part of the oxygen vacancy bar in Figure 1. Since the oxygen vacancy does not trap the positron, however, we expect that the lifetime will be reduced as the number of oxygen vacancies decrease, and therefore the lifetime associated with oxygen vacancies will approach the same value as the bulk, defect-free lifetime as the oxygen vacancy concentration becomes vanishingly small. This is indicated schematically in Figure 1 by the hatched region of the oxygen vacancy bar.

The right hand side of Figure 2 shows the change in the positron wavefunction on the vacancy site itself, compared with the wavefunction distribution for the bulk case. The change on the bismuth site is particularly dramatic. This is caused by the fact that the bismuth site is the least attractive site for the positron in the bulk case. The change on the oxygen vacancy site appears to be large, but this is produced by small changes on much larger atomic sites, and so has a much smaller overall effect on the lifetime than comparably sized changes on the potassium and barium sites.

If the sample contains a large number of oxygen vacancies, it is possible that the observed lifetime may be very much higher than the bulk, defect-free lifetime, but that the positron is not in a trapped state. This observation has important implications for 2DACAR measurements of Fermi surfaces[6]. It is important in such measurements that both the positron and the annihilating electron have well defined values of crystal momentum, $k$. The oxygen vacancies do not perturb the positron significantly, so its Bloch state is more or less unaltered. The electrons, of course, are more strongly affected, but the positron still annihilates with all the electrons in the solid, and those electrons trapped in oxygen vacancies will tend to contribute a wide spread of momenta, giving rise to a broad background term. The Fermi surface of interest may still be resolvable over this background. In contrast, the positron is strongly trapped at a metal vacancy site. In this case, it will not have a well defined value of momentum associated with it, so almost all of the annihilations will contribute to a broad, featureless background. Under such circumstances, it would be very difficult to detect Fermi surface features. Samples with lifetimes which are significantly longer than the bulk lifetime may therefore still be useful in Fermi surface studies using posicron annihilation, provided the increased lifetime is caused by oxygen vacancies.

\section{CONCLUSIONS}

First principles calculations of the positron lifetime in pure and defected $\mathrm{BKBO}$ have been used to analyse the experimental lifetime data of Ref. [3]. We find that the lifetime is dominated by annihilation at defect sites in all cases. The calculations strongly suggest 
that the $\mathrm{Ba}$ and $\mathrm{K}$ monovacancy is responsible for the positron trapping at higher temperatures, and in the aged $\mathrm{Ba}_{0.6} \mathrm{~K}_{0.1} \mathrm{BiO}_{3}$ sample. It is possible that the $\mathrm{Bi}$ vacancy, or some related defect structure, is responsible for the lower lifetimes observed. The temperature dependence of the lifetime has been interpreted in terms of a competition between these two states to trap the positron. The first principles calculations have provided us with a means of identifying the effects of defects on positron annihilation in these oxide superconductors.

The differences between the oxygen and metal vacancies will be apparent in many other systems besides BKBO. We expect that the insensitivity of the positron charge distribution to oxygen vacancies will be a common feature in many other metal oxides and oxide superconductors. In all cases, we expect the metal vacancies to provide effective trapping sites for the positrons, so care must be taken in interpreting positron annihilation data for these systems. By combining the interpretation of the experiments with theoretical calculations, such as those presented here, we can hope to resolve many of these problems.

\section{ACKNOWLEDGEMENTS}

Thir work was performed under the auspices of the U.S. Department of Energy by Lawrence Livermore National Laboratory under contract number W-7405-ENG-48. JHK acknowledges financial support from the Robert A. Welch Foundation, grant number Y'1135.

\section{REFERENCES}

[1] K. Petersen, in Positron Solid-State Physics edited by W. Brandt and A. Dupasquier (North Holland, Amsterdam, 1983), p. 298

[2] R. W. Siegel, in Positron Annihilation, edited by P. G. Coleman, S. C. Sharma and L. M. Diana (North-Holland, Amsterdam, 1982), p. 351

[3] J.C. O'Brien, R.H. Howell, H.B. Radousky, P.A. Sterne, D.G. Hinks, T.J. Folkerts and R.N. Shelton, in this volume.

[4] P.A. Sterne and J.H. Kaiser, submitted to Phys. Rev. B.

[5] H. L. Skriver, in The LMTO Method, Vol. 41 of Springer Series in Solid State Sciences (Springer, New York, 1984)

[6] R. N. West, Adv. Phys. 22, 263 (1973) 

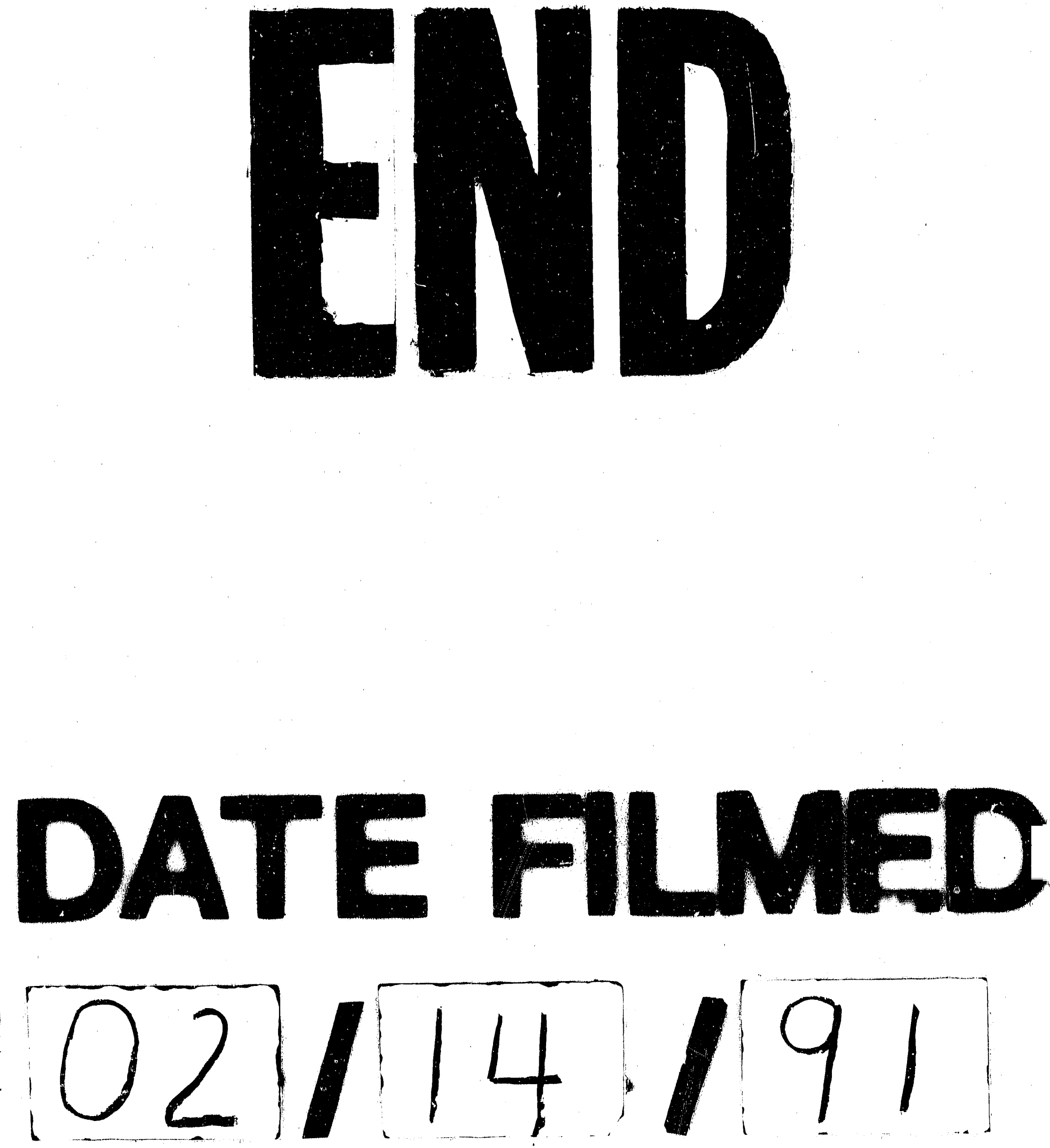
